International Journal of Pure and Applied Mathematics

Volume 84 No. 2 2013, 39-48

ISSN: 1311-8080 (printed version); ISSN: 1314-3395 (on-line version)

url: http://www.ijpam.eu

doi: http://dx.doi.org/10.12732/ijpam.v84i2.3

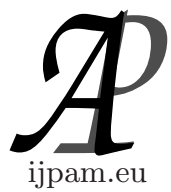

\title{
EXCESSIVE INDEX OF CERTAIN CHEMICAL STRUCTURES
}

\author{
Paul Manuel ${ }^{1}$, Indra Rajasingh ${ }^{2}$, R. Bharati ${ }^{3}$, A.S. Shanthi ${ }^{4} \S$ \\ ${ }^{1}$ Department of Information Science \\ Kuwait University \\ Safat, KUWAIT \\ ${ }^{2}$ School of Advanced Sciences \\ VIT University \\ Chennai, 600 127, INDIA \\ ${ }^{3,4}$ Department of Mathematics \\ Loyola College \\ Chennai, 600 034, INDIA
}

\begin{abstract}
The structural formulae of chemical compounds are molecular graphs where vertices represent atoms and edges represent chemical bonds. A kekule structure in a molecular graph is nothing but a perfect matching in the graph. The minimum number of kekule structures that cover the edge set of a molecular graph $G$ is known as the excessive index of $G$. In this paper we determine the excessive index of the unit cell representation of sodium chloride and 3- $D$ mesh networks representing oxides and selenides of certain chemical compounds. Further we determine the excessive index of hexagonal networks.
\end{abstract}

AMS Subject Classification: 05C70, 05C45

Key Words: matching, perfect matching, excessive index, hexagonal networks, 3- $D$ mesh network

Received: October 4, 2012

(c) 2013 Academic Publications, Ltd. url: www.acadpubl.eu

${ }^{\S}$ Correspondence author 


\section{Introduction}

Mathematical chemistry has been formally recognized only during the last few decades, with a strong influence involving graph theoretic work. This special influence is perhaps quite appropriate in view of its relevance in the classical core of chemistry, the characterization of molecular structure, and valence theory [18]. Chemical graph theory models have been extensively used as predictors of the properties of chemical compounds. All structural formulae of chemical compounds are molecular graphs where vertices represent atoms and edges represent chemical bonds.

The study of Kekule structures of chemical compounds have many hidden treasures [22] and have for a long time been the focus of interest of scholars working on the theory of benzenoid molecules [14]. A vast amount of theoretical work also has been done on Kekule structures [10, 11, 12, 13]. Carbon compounds without kekule structures are considered in [22].

One major direction of research is finding the number $K$ of Kekule structures [7] and trying to relate $K$ with various physico-chemical properties of the underlying compounds [15]. Another direction is the study of individual Kekule structures and finding relations between them. A famous problem along these lines which has not been satisfactorily solved is the identification of the Kekule structure that provides the most faithful representation of the true bonding in the respective molecule [17].

A kekule structure in a molecular graph is nothing but a perfect matching in the graph. The minimum number of kekule structures (perfect matchings) that cover the edge set of a molecular graph $G$ (or simply a graph $G$ ) is known as the excessive index of $G[2]$.

Excessive index has a number of applications particularly in scheduling theory to complete a process in the minimum possible time [5]. Scheduling is important in modern production and chemical industries, where it can have a major impact in the productivity of a process. In this paper we determine the excessive index for hexagonal and $3-D$ mesh network.

\section{Preliminaries}

A matching in a graph $G=(V, E)$ is a subset $M$ of edges, no two of which have a vertex in common. A matching $M$ is said to perfect if every vertex in $G$ is an endpoint of one of the edges in $M$. Thus a perfect matching in $G$ is a 1-regular spanning subgraph of $G$. In the literature it is also known as a 1- 


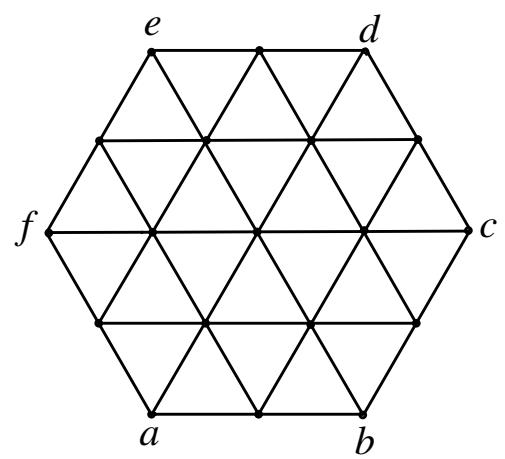

Figure 1: Hexagonal Network of dimension 3

factor of $G$. A near-perfect matching covers all but exactly one vertex. A graph $G$ is 1-extendable if every edge of $G$ belongs to at least one 1-factor of $G$. A 1 -factor cover of $G$ is a set $\mathcal{F}$ of 1 -factors of $G$ such that $\cup_{F \in \mathcal{F}} F=E(G)$. A 1factor cover of minimum cardinality is called an excessive factorization [2]. An excessive near 1-factorization of a graph $G$ is a minimum set of near 1-factors whose union contains all the edges of $G$ [4].

The excessive index of $G$, denoted $\chi_{e}^{\prime}(G)$, is the size of an excessive factorization of $G$. We define $\chi_{e}^{\prime}(G)=\infty$ if $G$ is not 1-extendable. A graph $G$ is 1-factorizable if its edge set $E(G)$ can be partitioned into edge-disjoint 1-factors. The problem of determining whether a regular graph $G$ is 1-factorizable is $N P$ complete [16]. Bonisoli et al. [2] observed that the problem of determining the excessive index for regular graphs is $N P$-hard. Cariolaro et al. [3] determined the excessive index of complete multipartite graphs, which proved to be a challenging task. The excessive index of a bridgeless cubic graph has been studied by Fouquet et al. [9]. Excessive index of certain regular graphs has been determined in $[1,20]$.

\section{Hexagonal Networks}

Hexagonal networks are based on regular triangular tessellations, or the partition of a plane into equilateral triangles and are widely studied in [6]. They are applied in chemistry to model benzenoid hydrocarbons [23], in image processing \& computer graphics [19], and wireless \& interconnection networks. Hexagonal network $H X(n)$ of dimension $n$ has $3 n^{2}-3 n+1$ vertices and $9 n^{2}-15 n+6$ edges, where $n$ is the number of vertices on one side of the hexagon [6]. See Figure 1 . The diameter is $2 n-2$. There are six vertices of degree three which we call 


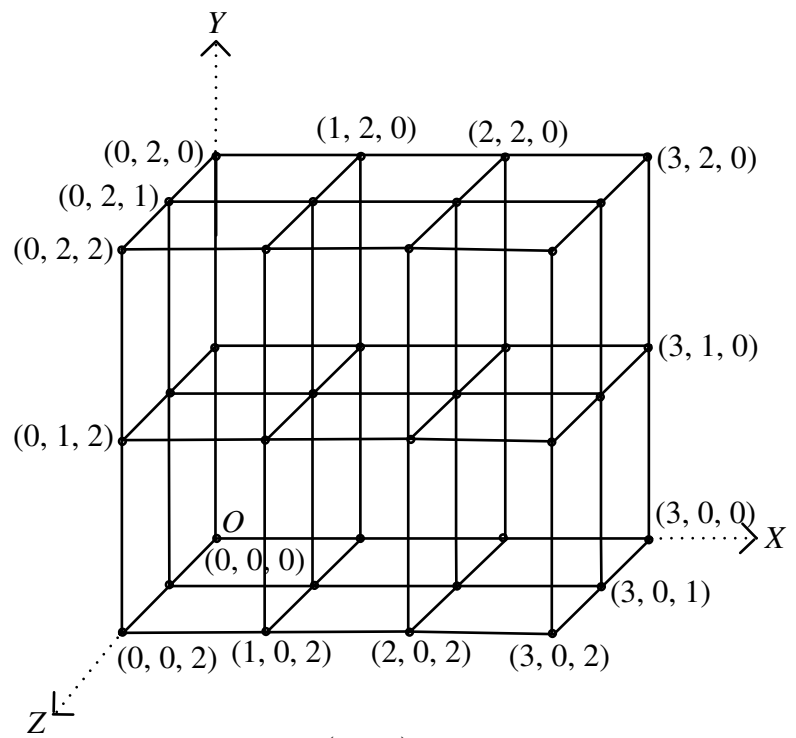

Figure 2: $(3-D)$ mesh $M_{4 \times 3 \times 3}$

as corner vertices. Name the corner vertices as $a, b, c, d, e$ and $f$ as shown in Figure 1. The performance of hexagonal networks was studied in $[6,8,21]$.

Theorem 3.1. [2] Let $G$ be a graph. Then $\chi_{e}^{\prime}(G) \geq \triangle$.

In view of Theorem 3.1, we have the following theorem.

Theorem 3.2. Let $G$ be the hexagonal network $H X(n)$ of dimension $n$. Then $\chi_{e}^{\prime}(G) \geq 6$.

We now give a procedure and its proof of correctness to show that the lower bound obtained in Theorem 3.2 is sharp.

Procedure Excessive Index $\chi_{e}^{\prime}(H X(n))$

Input: The hexagonal network $H X(n)$ of dimension $n$.

\section{Algorithm:}

Choose $a b$ as the base line. See Figure 1 . We note that $a$ and $d$ are diametrically opposite vertices. We construct a hamiltonian path $P_{a d}$ in $H X(n)$ from $a$ to $d$ as follows: 
Traverse the base line starting from $a$ and all lines parallel to the base line with appropriate oblique boundary edges connecting these parallel lines. Let $M_{1}$ consist of alternate edges on $P_{a d}$ starting from the edge with one end at $a$. In $M_{2}$ choose edges on $P_{a d}$ which are not in $M_{1}$. Let $M_{3}$ and $M_{4}$ be similarly formed by choosing the hamiltonian path $P_{b e}$ with $b c$ as the base line. Further $M_{5}$ and $M_{6}$ are obtained in a similar manner by choosing the hamiltonian path $P_{c f}$ with $c d$ as the base line.

End Excessive Index $\chi_{e}^{\prime}(H X(n))$

Output: $\quad \chi_{e}^{\prime}(H X(n))=6$.

Proof of Correctness. The edges of hexagonal network are in three different direction and each of the hamiltonian paths $P_{a d}, P_{b e}$ and $P_{c f}$ covers those edges. The result follows from the observation thus $P_{a d}=M_{1} \cup M_{2}, P_{b e}=M_{3} \cup M_{4}$ and $P_{c f}=M_{5} \cup M_{6}$.

Theorem 3.3. Let $G$ be the hexagonal network $H X(n)$ of dimension $n$. Then $\chi_{e}^{\prime}(G)=6$.

\section{Mesh Networks}

Let $P_{n}$ denote a path on $n$ vertices. For $m, n \geq 2, P_{m} \times P_{n}$ is defined as a two dimensional (2-D) mesh with $m$ rows and $n$ columns. A three dimensional (3- $D$ ) mesh $M_{r \times s \times t}$ is nothing but $P_{r} \times P_{s} \times P_{t}$. See Figure 2. In a 3-D mesh there are str number of vertices and $(2 r s-r-s) t+(t-1) s r$ number of edges. In our day-to-day life, the common salt $\mathrm{NaCl}$ is used as an important preservative because it retards the growth of micro-organisms. It also improves the flavour of food items. Chlorine products are used in metal cleaners, paper bleach, plastics and water treatment. They are also used in medicines. We find that the unit cell representation of Sodium Chloride $(\mathrm{NaCl})$ is the same as the $3-D$ mesh $M_{3 \times 3 \times 3}$. See Figure 3 .

In the $3-D$ mesh $M_{3 \times 3 \times 3}$, in the place of chlorine $C l$ if we take iron and in the place of sodium if we take oxygen it is nothing but the crystal structure of iron oxide. Similarly sodium replaced by magnesium and chloride replaced by selenide is the crystal structure of magnesium selenide. Further the crystal structure of cobalt oxide, magnesium oxide, europium selenide, lead selenide is the 3 - $D$ mesh $M_{3 \times 3 \times 3}$. For convenience we shall make use of the cartesian coordinate system for the $3-D$ mesh. Let the mutually perpendicular lines through $O$ be the $X$-axis, $Y$-axis and $Z$-axis respectively. The vertices of $M_{r \times s \times t}$ are 


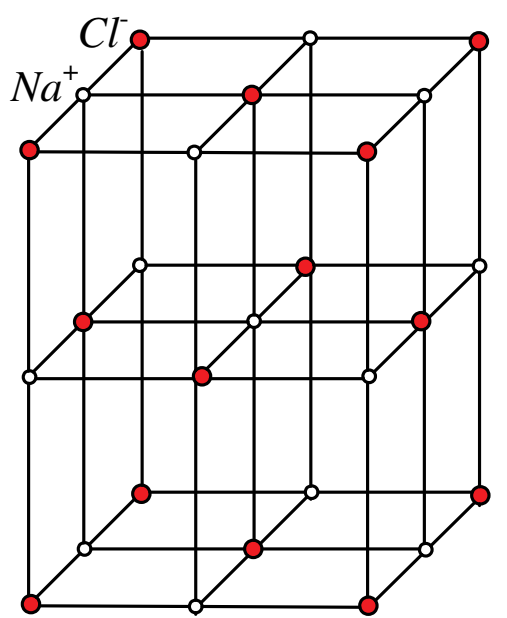

Figure 3: Sodium Chloride ( $\mathrm{NaCl})$

denoted by $(i, j, k), 0 \leq i \leq r-1,0 \leq j \leq s-1,0 \leq k \leq t-1$ and the edge joining two vertices $(a, b, c)$ and $(d, e, f)$ is denoted by $((a, b, c),(d, e, f))$. The vertex $O$ is represented as $(0,0,0)$ and the remaining vertices are labeled as shown in the Figure 2.

Theorem 4.1. Let $G$ be the mesh network $M_{r \times s \times t}$. Then $\chi_{e}^{\prime}(G)=6$.

By Theorem 3.1, $\chi_{e}^{\prime}(G) \geq 6$.

Case (i): $r$ even.

In $M_{1}$ select the edges $((i, j, k),(i+1, j, k)), i$ even, $0 \leq i \leq r-2$, $0 \leq j \leq s-1,0 \leq k \leq t-1$. Clearly $M_{1}$ is perfect. In $M_{2}$ select the edges $((i$, $j, k),(i+1, j, k)), i$ odd, $1 \leq i \leq r-3,0 \leq j \leq s-1,0 \leq k \leq t-1$. Since $r$ is even, the vertices $(i, j, k), i=0, r-1,0 \leq j \leq s-1,0 \leq k \leq t-1$ are unsaturated. To saturate these vertices, select the edges $((i, j, k),(i, j+1, k))$, $i=0, r-1 j$ even, $0 \leq j \leq s-2,0 \leq k \leq t-1$.

If $s$ is even then $M_{2}$ is perfect. See Figure 4. If $s$ is odd the vertices $(i$, $s-1, k), i=0, r-1, j=s-1,0 \leq k \leq t-1$ are left unsaturated. In order to saturate these vertices, select the edges $((i, s-1, k),(i, s-1, k+1)), i=0, r-1$, $k$ even, $0 \leq k \leq t-2$. If $t$ is even then $M_{2}$ becomes perfect. If $t$ is odd the vertices $(0, s-1, t-1)$ and $(r-1, s-1, t-1)$ are left unsaturated. In order to saturate these vertices, consider the vertices $(i, s-1, t-1), 0 \leq i \leq r-1$. The edges induced by the vertices $(i, s-1, t-1)$ and $(i+1, s-1, t-1), i$ odd, $1 \leq i \leq r-3$ are already in $M_{2}$. Now remove these selected edges from $M_{2}$ and include the edges $(i, s-1, t-1)$ and $(i+1, s-1, t-1), i$ even, $0 \leq i \leq r-2$ 
in $M_{2}$. Now $M_{2}$ is perfect.

Case (ii): $r$ odd.

In $M_{1}$ select the edges $((i, j, k),(i+1, j, k)), i$ even, $0 \leq i \leq r-3$, $0 \leq j \leq s-1,0 \leq k \leq t-1$. Since $r$ is odd, the vertices $(r-1, j, k)$, $0 \leq j \leq s-1,0 \leq k \leq t-1$ are unsaturated. To saturate these vertices, select the edges $((r-1, j, k),(r-1, j+1, k)), j$ even, $0 \leq j \leq s-2,0 \leq k \leq t-1$. If $s$ is even then $M_{1}$ is perfect. If $s$ is odd the vertices $(r-1, s-1, k), 0 \leq k \leq t-1$ are unsaturated. In order to saturate these vertices, select the edges $((r-1$, $s-1, k),(r-1, s-1, k+1)), k$ even, $0 \leq k \leq t-2$. If $t$ is even then $M_{1}$ becomes perfect. If $t$ is odd $M_{1}$ becomes near-perfect, since there are odd number of vertices in $M_{r \times s \times t}$ when $r, s$ and $t$ are all odd.

In $M_{2}$ select the edges $((i, j, k),(i+1, j, k)), i$ odd, $1 \leq i \leq r-2$, $0 \leq j \leq s-1,0 \leq k \leq t-1$. Since $r$ is odd, the vertices $(0, j, k), 0 \leq j \leq s-1$, $0 \leq k \leq t-1$ are left unsaturated. Repeat the procedure as in $M_{1}$ when $(r-1$, $j, k), 0 \leq j \leq s-1,0 \leq k \leq t-1$ are unsaturated. Thus in $M_{1}$ and $M_{2}$ we consider the edges parallel to $X$-axis and to make them perfect if necessary we consider the edges parallel to $Y$-axis then $Z$-axis. We denote this process by $X \longrightarrow Y \longrightarrow Z$. In $M_{3}$ and $M_{4}$, repeat the procedure with $Y \longrightarrow Z \longrightarrow X$ and in $M_{5}$ and $M_{6}$ with $Z \longrightarrow X \longrightarrow Y$.

Thus $M_{1} \cup M_{2}$ covers all edges parallel to $X$-axis except when $r$ is even and $s$ and $t$ are odd. In this case in order to cover $((i, s-1, t-1),(i+1, s-1$, $t-1)), i$ odd, $1 \leq i \leq r-3$ edges parallel to $X$-axis, remove the edges parallel to $Z$-axis as well as edges parallel to $X$-axis in $M_{3}$ and include the edges $((i$, $s-1, k),(i+1, s-1, k)), i$ odd, $1 \leq i \leq r-3,1 \leq k \leq t-1,((i, s-1, k),(i$, $s-1, k+1)), i=0, r-1, k$ odd, $1 \leq k \leq t-2$ and $((i, s-1,0),(i+1, s-1$, $0)), i$ even, $0 \leq i \leq r-2$.

\section{Conclusion}

In this paper we have determined the excessive index of hexagonal and 3- $D$ mesh networks yielding the excessive index of sodium chloride, cobalt oxide, magnesium oxide, europium selenide, lead selenide and many more. It would be an interesting line of research to determine the excessive index for other chemical structures since scheduling theory has gained momentum in biotechnology and even in nanotechnology. 


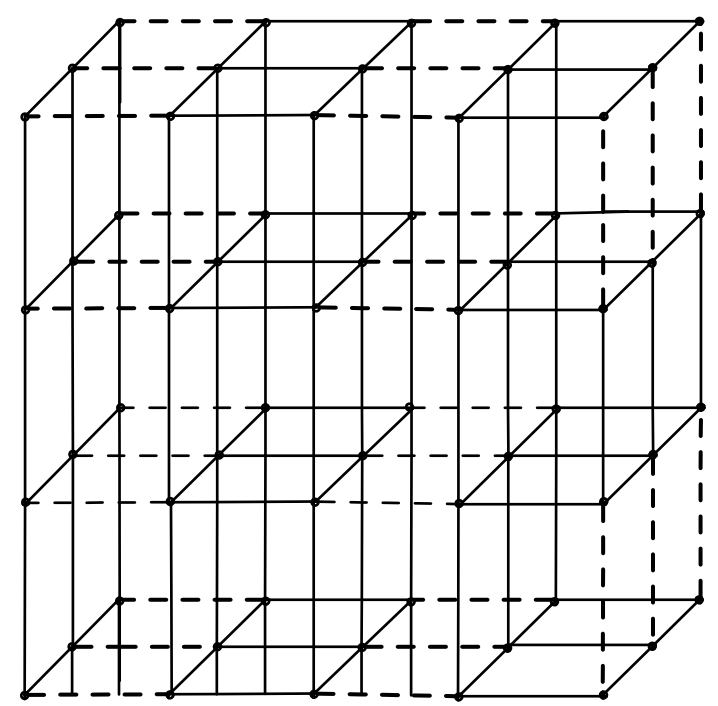

Figure 4: Dotted lines shows the edges selected in $M_{1}$ of $M_{5 \times 4 \times 3}$

\section{References}

[1] S. Belcastro and M. Young, 1-factor covers of regular graphs, Discrete Applied Mathematics, 159, No. 5 (2011), 281-287.

[2] A. Bonisoli and D. Cariolaro, Excessive factorizations of regular graphs, In: Graph Theory in Paris, Birkhauser, Basel, (2007), 73-84.

[3] D. Cariolaro and H.L. Fu, On minimum sets of 1-factors covering a complete multipartite graph, Journal of Graph Theory, 58, No. 3 (2008), 239250 .

[4] D. Cariolaro and H.L. Fu, Excessive near 1-factorizations, Discrete Mathematics, 309, No. 14 (2009), 4690-4696.

[5] D. Cariolaro and H.L. Fu, The excessive 3-index of all graphs, Electronic Journal of Combinatorics, 16, No. 1 (2009), Research Paper 124.

[6] M.S. Chen, K.G. Shin and D.D. Kandlur, Addressing, routing, and broadcasting in hexagonal mesh multiprocessors, IEEE Transactions on Computers, 39, (1990), 10-18.

[7] S.J. Cyvin and I. Gutman, Kekule Structures in Benzenoid Hydrocarbons, Springer-Verlag, Berlin, (1988). 
[8] J.W. Dolter, P. Ramanathan and K.G. Shin, Performance analysis of virtual cut-through switching in HARTS: A hexagonal mesh multicomputer, IEEE Transactions on Computers, 40, (1991), 669-680.

[9] J.L. Fouquet and J.M. Vanherpe, On the perfect matching index of bridgeless cubic graphs, CoRR abs/0904.1296, (2009).

[10] K. Friess, Liebigs Ann. Chem., 454, (1927), 121.

[11] K. Friess, R. Walter and K. Schilling, Liebigs Ann. Chem., 516, (1935), 248.

[12] A. Graovac, I. Gutman, M. Randic and N. Trinajstic, Journal of the American Chemical Society, 95, (1973), 6267.

[13] I. Gutman and O. Polansky, Mathematical Concepts in Organic Chemistry, Springer-Verlag, Berlin, (1986).

[14] I. Gutman, B. Arsic, M. Denic and I. Stojanovic, Benzenoid isomers with greatest and smallest Kekule structure counts, Journal of the Serbian Chemical Society, 71, No. 7 (2006), 785-791.

[15] I. Gutman and S.J. Cyvin, Introduction to the theory of benzenoid hydrocarbons, Springer-Verlag, Berlin, (1989).

[16] I. Holyer, The NP-completeness of edge colouring, SIAM Journal on Computing, 10, No. 4 (1981), 718-720.

[17] S. Klavzar, A. Vesel, P. Zigert and I. Gutman, Binary coding of Kekule structures of catacondensed benzenoid hydrocarbons, Computers chemistry, 25, No. 6 (2001), 569-575.

[18] D.J. Klein and A. Misra, Minimally kekulenoid-Networks and reactivity for acyclics, Croatica Chemica ACTA CCACAA, 77, No. (1-2) (2004), 179-191.

[19] E. Kranakis, H. Singh and J. Urrutia, Compass routing in geometric graphs, Proceedings of the 11th Canadian Conference on Computational Geometry, (CCCG-99), (1999), 51-54.

[20] G. Mazzuoccolo and M. Young, Graphs of arbitrary excessive class, Discrete Mathematics, 311, No. 1 (2011), 32-37. 
[21] G. Rote, On the connection between hexagonal and undirected rectangular systolic arrays, Lecture Notes in Computer Science, Springer-verlag, 227, (1986), 70-83.

[22] S. Tang and H. Deng, On the anti-kekule number of three fence graphs, Digest Journal of Nanomaterials and Biostructures, 6, No. 2 (2011), 439443.

[23] R. Tosic, D. Masulovic, I. Stojmenovic, J. Brunvoll, B.N. Cyvin, and S.J. Cyvin, Enumeration of polyhex hydrocarbons up to $h=17$, Journal of Chemical Information and Computer Sciences, 35, (1995), 181-187. 